\title{
On the Fabry-Ehrenpreis-Kawai Gap Theorem
}

\author{
By \\ Carlos A. Berenstein* and Daniele C. Struppa**
}

\section{§. Introduction}

The classical Fabry gap theorem, see e. g. [5], can be formulated, after an exponential change of variables, as follows:

Theorem 0. Let $\sum_{i=1}^{+\infty} a_{j} \exp (i \cdot j \cdot z), z \in \boldsymbol{C}$, be a series which converges, uniformly on the compact subsets of $\Pi_{+}=\{z \in \mathbb{C}: \operatorname{Im} z>0\}$, to an analytic function $f(z)$. Suppose, moreover, that $f$ extends analytically to $\{z \in \mathbb{C}:|z|<\delta\}$, for some $\delta>0$, and that $a_{\jmath}=0$ except for $j$ in a subsequence $\left\{b_{k}\right\}$ such that the number of $b_{k}$ smaller than $N$ is $o(N)$, for $N \rightarrow \infty$. Then $f(z)$ extends analytically to $\Pi_{+}-i \delta$ $=\{z \in C: \operatorname{Im} z>-\delta\}$, and the above series converges to $f$ on the compact subsets of this set.

In his book [2], Ehrenpreis gave a completely new treatment of this theorem, which is based on his theory of Analytically Uniform spaces, and on the observation that each function $\exp (i \cdot b, \cdot z)$ can be thought of as a solution of the simple differential equation

$$
\frac{d f}{d z}-i \cdot b_{\jmath} \cdot f=0
$$

therefore, a natural generalization (which is the one considered by Ehrenpreis) consists in looking at series of the form $\sum_{j=1}^{+\infty} f_{j}$, for the $f_{j}$ solutions of suitable (systems of) differential equations.

A different approach which is suggested, but not pursued, by Ehrenpreis, consists in thinking of $\sum a_{j} \exp (i \cdot b, z)$ as the solution of a suitable convolution equation $\mu * f=0$, for $f \in \mathscr{H}\left(\Pi_{+}\right), \mu \in \mathscr{H}^{\prime}(\mathbb{C})$ ( $\mathscr{H}$ and $\mathscr{H}^{\prime}$ denote, respectively, the spaces of holomorphic functions and of analytic functionals). This point of view was considered by Kawai in [3], who provided a genuine extension of Ehrenpreis' results, with the use of completely different techniques, based on

Communicated by M. Kashiwara, January 9, 1987.

Department of Mathematics, University of Maryland, College Park, Maryland 20742, U.S.A. and Scuola Normale Superiore, Piazza dei Cavalieri, 7, 56100 Pisa, Italy.

* The Research of the first author was supported in part by the National Science Foundation.

** Present address : Dipartimento di Matematica, Universita della Calabria, Arcavacata di Rendo (CS), Italy. 
the theory of holomorphic microlocal operators as described in [8]; in his paper, therefore, Kawai is able to considerably extend the Fabry gap theorem, by considering convolution operators which are determined by suitable hyperfunctions supported at the origin.

In this article, on the other hand, we employ convolution operators defined by suitable analytic functionals, to prove a different extension (Theorem 1) of the Fabry gap theorem. Our techniques are more classical (in spirit) and are based on some recent works on convolution equations spaces of holomorphic functions, [1], [6]. Some restrictions are needed on the analytic functionals, but we provide large classes of examples to which our theorem applies (Proposition 1 and Remarks 3,4).

Finally, we combine our result with Kawai's one to provide a further extension (Theorem 2) which applies to the case of convolutors which can be factorized into a slowly decreasing one (see Definition 1 below) and into a suitable differential operator of infinite order.

The paper is concluded by a short remark on the situation in $C^{n}, n>1$.

We wish to express our gratitude to the Scuola Normale Superiore for inviting the first author, during the period in which this paper was written.

\section{§2. Convolution Equations}

This is a preliminary section in which we provide the necessary background information on convolution equations in spaces of holomorphic functions.

Let $\mu \in \mathscr{K}^{\prime}(\boldsymbol{C})$ be an analytic functional, carried by some compact convex set $K \subset C$, and let $\Omega$ denote an open convex set in $C$. Then $\mu$ acts as a convolutor on $\mathscr{H}(\Omega+K)$ as follows:

$$
\begin{aligned}
\mu *: \mathscr{H}(\Omega+K) \longrightarrow & \mathscr{H}(\Omega) \\
f & \longrightarrow \mu * f(z):=\langle\mu, \xi \rightarrow f(z+\xi)\rangle .
\end{aligned}
$$

As it is well known, the properties of this convolutor are reflected in the properties of the Fourier-Borel transform of $\mu$, namely

and the spaces

$$
\hat{\mu}(z):=\langle\mu, \xi \rightarrow \exp (z \cdot \xi)\rangle,
$$

$$
\begin{array}{r}
\mathscr{H}^{\prime}(\Omega) \cong\{F \in \mathscr{H}(\mathbb{C}): \exists A>0, T \subset \Omega \quad \text { compact, such that, } \forall z \in C, \\
\left.|F(z)| \leqq A \exp \left(H_{T}(z)\right)\right\} \\
\mathscr{H}^{\prime}(\Omega+K) \cong\{F \in \mathscr{H}(\boldsymbol{C}): \exists A>0, T \subset \Omega \quad \text { compact, such that, } \forall z \in \mathbb{C}, \\
\left.|F(z)| \leqq A \exp \left(H_{T+K}(z)\right)\right\} ;
\end{array}
$$


here and in the sequel, for $T$ a compact set, $H_{T}(z):=\sup _{\Sigma} \subseteq T \operatorname{Re}(z \cdot \bar{\xi})$ denotes the supporting function of the set $T$.

Remark 1. The isomorphisms between $\mathscr{H}^{\prime}(\Omega), \mathscr{H}^{\prime}(\Omega+K)$ and the corresponding spaces of entire functions with growth control, are given by the Fourier-Borel transform, on the other hand, if $\mu \in \mathscr{C}^{\prime}(\mathbb{C})$ is carried by $K$, then $\forall \varepsilon>0 \exists A_{\varepsilon}>0$ such that

$$
|\hat{\mu}(z)| \leqq A_{\varepsilon} \exp \left(H_{K}(t)+\varepsilon|z|\right) .
$$

In order to prove our extension of the Fabry gap theorem, we need to restrict our attention to a special class of analytic functionals: from [6] we quote

Definition 1. The analytic functional $\mu \in \mathcal{H}^{\prime}(\mathbb{C})$ is said to be slowly decreasing if, for $V=\{z \in \mathbb{C}: \hat{\mu}(z)=0\}, K$ a carrier of $\mu$, and $d(z, V)=$ $\min (1, \operatorname{dist}(z, V))$, the following conditions holds :

(i) $\exists m$ integer such that and $\forall \varepsilon>0 \exists C_{\varepsilon}>0$ so that

$$
|\hat{\mu}(z)| \geqq C_{\varepsilon}(d(z, V))^{m} \exp \left(H_{K}(z)+\varepsilon|z|\right)(1+|z|)^{-m}, \quad z \subseteq C ;
$$

(ii) $\forall \varepsilon>0 \exists A_{\varepsilon}>0$ such that the set

$$
\left\{z \in \mathbb{C}: d(z, V) \leqq A_{\varepsilon} \exp (-\varepsilon|z|)\right\}
$$

has relatively compact connected components, of uniformly bounded diameters.

A few remarks are necessary to clarify the meaning of this condition.

Remark 2. One of the main problems in the theory of convolution equations is to establish whether every solution of $\mu * f=0$ admits a representation as a convergent series of "elementary" solutions. This is not true in general, and extra conditions on $\mu$ are usually necessary. Theorem 7 of [6] shows that if $\mu$ is slowly decreasing, then one can actually obtain the series representation. To this purpose, the reader might notice that (i) is slightly weaker than the condition required in [6]: nevertheless, if one follows through the arguments employed in [6] to establish Theorem 7, one can show that our Definition 1 is still sufficient to recover the representation results.

Remark 3. As Definition 1 is rather complicated, one might wonder on the existence of examples for it. Indeed in [6] (but, essentially, already in [1]), it is mentioned that exponential polynomials, i.e. functions like $\sum_{\imath=1}^{N} c_{\jmath}(z) \exp \left(i \cdot a_{\jmath} \cdot z\right)$, $a_{\jmath} \in \mathbb{C}, c_{\jmath}(z) \in \mathbb{C}[z]$, do indeed satisfy the conditions of Definition 1 (in terms of convolution equations, this simply means that our theory works for differencedifferential equations). 
Remark 4. A further simplification in Definition 1 has been recently established by Yger [10]. Indeed (Yger works in a slightly different space of functionals but his arguments apply to our situation as well) he proved that, provided that the zeroes of $\hat{\mu}$ do not coalesce too quickly asymptotically ( $V$ is well-separated) then (i) actually implies (ii). The reader is referred to [10], remarque 1.2 , for further details.

In order to relate Definition 1 with more common concepts in the theory of entire functions, we introduce some other definitions.

Definition 2. Let $f \in \mathscr{H}(\boldsymbol{C})$ be an entire function, $f \not \neq 0$; its multiplicity variety $V(f)$ is the set of pairs $\left(z_{k}, m_{k}\right), z_{k} \in C, m_{k} \geqq 1$ integer, where $z_{k}$ runs over all the zeroes of $f$, and $m_{k}$ denotes the multiplicity of that zero.

Definition 3. Let $V=\left\{\left(z_{k}, m_{k}\right)\right\}$ be a multiplicity variety, and let $\Omega$ be an open convex subset of $\boldsymbol{C}$. We say that $V$ is $\Omega$-interpolating if for every sequence $\left\{a_{k, l}\right\}_{k \in \boldsymbol{N}, 0 \leqq l<m_{k}}$ such that

$$
\sum_{l=0}^{m_{k}-1}\left|a_{k, l}\right| \leqq A \exp \left(H_{T}\left(z_{k}\right)\right)
$$

for some $A>0$ and some compact $T \subset \Omega$, there exists an entire function $F \in \hat{\mathcal{H}}^{\prime}(\Omega)$ such that $\left(d^{l} F / d z^{l}\right)\left(a_{k}\right)=l ! a_{k, l}, 0 \leqq l<m_{k}, \quad k=1,2, \cdots$

Remark 5. If the multiplicities $m_{k}$ are bounded, (2) is equivalent to

$$
\left|a_{k, l}\right| \leqq A \exp \left(H_{T}\left(z_{k}\right)\right) \text {. }
$$

Remark 6. Condition (ii) in Definition 1 holds automatically if $\{\hat{\mu}=0\}$ is an interpolating variety. The details of the argument can be found in [9].

Definition 4. Let $f(z)$ be an entire function of exponential type. We say that $f$ is of completely regular growth if, $\forall \theta \in[0,2 \pi]$, the limit

$$
\Lambda_{f}(\theta)=\lim _{r \rightarrow+\infty} \ln \left|f\left(r e^{i \theta}\right)\right| \cdot r^{-1}
$$

exists when $r$ goes to infinity by taking on all positive values, except possibly for a set $E_{\theta}$ of zero relative measure, this set being the same for all values of $\theta$.

Functions of completely regular growth are frequently met for several different reasons. In the theory of entire functions the property we are interested in is expressed by the following lemma, essentially due to Morzhakov [7]:

Lemma 1. Let $f=\hat{\mu}$ be the Fourier-Borel transform of an analytic functional $\mu \in \mathcal{H}^{\prime}(\boldsymbol{C})$, carried by a compact $K$, and let $\Omega \subset C$ be open and convex. Suppose $f$ is of completely regular growth: then, for every $g \in \hat{\mathcal{H}}^{\prime}(\Omega+K)$, if $g / f \in \mathscr{H}(\boldsymbol{C})$, 
then $g / f \in \hat{\mathcal{K}}^{\prime}(\Omega$. In particular, the convolution operator $\mu *: \mathscr{H}(\Omega+K) \rightarrow \mathscr{H}(\Omega)$ is surjective.

Proof. Let $g / f=h \in \mathscr{H}(\boldsymbol{C})$. By the hypothesis on $f$ and [4], corollary 2 page 160 , one has $\Lambda_{h}(\theta)=\Lambda_{g}(\theta)-\Lambda_{f}(\theta)$. Since $g \in \hat{\mathscr{H}}^{\prime}(\Omega+K), f=\hat{\mu}, \quad \mu$ carried by $K$, one has $\Lambda_{g}(\theta) \leqq H_{K+K_{1}}\left(e^{i \theta}\right)$ for some compact $K_{1} \subset \Omega$, and $\Lambda_{f}(\theta)=H_{K}\left(e^{i \theta}\right)$, i. e.

$$
\Lambda_{l l}(\theta) \leqq H_{K_{1}}\left(e^{i \theta}\right),
$$

which shows that $h \in \hat{\mathcal{H}}^{\prime}(\Omega)$. The surjectivity of $\mu *$ is now an immediate consequence of a standard argument in functional analysis: $\mu *$ is onto iff its adjoint $\mu^{* \prime}: \mathscr{H}^{\prime}(\Omega) \rightarrow \mathscr{H}^{\prime}(\Omega+K)$ is injective and of closed range. By applying the Fourier-Borel transform, this follows from the first part of the lemma.

Remark 7. In [7] it is shown that the converse is true as well: if $\mu *$ is onto, then $\hat{\mu}$ must be of regular growth. We refer the reader to $[6]$ for further details on this matter.

Remark 8. If $\mu$ is slowly decreasing as in Definition 1 , then $\mu *$ is surjective, and therefore $\hat{\mu}$ is of completely regular growth.

The following weaker property still implies the surjectivity of $\mu *$ :

Definition 5. Let $f$ be an entire function of exponential type. We say that $f$ is $K$-invertible, for $K \subset \mathbb{C}$ a compact convex set, if $\forall s>0$ there is a constant $A_{\varepsilon}>0$ such that the set

$$
\left\{z \in \mathbb{C}:\left|f^{\prime}(z)\right|<A_{\varepsilon} \exp \left(H_{K}(z)-\varepsilon|z|\right)\right\}
$$

has relatively compact connected components, of uniformly bounded diameters.

Before proving that, for large classes of entire functions, (1) always holds, we prove a "local" version of the same result:

Lemma 2. Let $\mu \in \mathscr{H}^{\prime}(\mathbb{C})$, be carried by a compact convex sii $K$, sat $f=\hat{\mu}$ and let $\Omega$ bc an opcn and convex subset of $\mathbb{C}$. If $V=V(f)=\left\{\left(z_{k}, m_{k}\right)\right\}$ is $(\Omega+K)$-interpolating and if $f$ is K-invertibie (or, more gcnerally, of comtletely reguvar growth), thein $\forall \varepsilon>0 \exists C_{\varepsilon}>0$ such that

$$
\left|f^{\left(m_{k}\right)}\left(z_{k}\right)\right| \geqq C_{\varepsilon} m \eta_{k} ! \exp \left(H_{r_{i}}\left(z_{k}\right)-\varepsilon\left|z_{k}\right|\right) \text {. }
$$

Proof. First notice that it is possible to find a constant $C>0$, a compact $T=K_{1}+K \subset \Omega+K$ (for $K_{1} \subset \Omega$ compact), and entire functions $h_{k}$ such that

$$
\left|h_{k}(z)\right| \leqq C \exp \left(h_{T}^{r}(\boldsymbol{z})\right),
$$

and, for ali $\left(z_{j}, m_{\jmath}\right) \in V$, 


$$
h_{k}^{(l)}\left(z_{j}\right)=0 \quad 0 \leqq l<m_{\jmath},
$$

unless $j=k$ and $l=m_{k}-1$, when

$$
h_{k}^{\left(m_{k}-1\right)}\left(z_{k}\right)=\left(m_{k}-1\right) ! \exp \left(H_{K+K_{2}}\left(z_{k}\right)\right),
$$

for some compact $K_{2} \subset \Omega$. This is, indeed, a consequence of the open mapping theorem and of the interpolating property of $V(f)$ (see also [1], Theorem 4). Now, $\left(z-z_{k}\right) h_{k} / f$ is entire and, by the $K$-invertibility of $f$, we get

$$
\left(z-z_{k}\right) h_{k}=f \cdot g_{k},
$$

where the entire function $g_{k}$ satisfies $\forall \varepsilon>0$ the estimate

$$
\left|g_{k}(z)\right| \leqq A_{\varepsilon} \exp \left(H_{K_{1}}(z)+\varepsilon|z|\right),
$$

for some $A_{\varepsilon}>0$. The lower bound (3) now follows by equating the leading terms of the power series expansion about $z_{k}$ of both sides of (4), and using the upper bound on the $g_{k}$ given in (5).

This lemma is sufficient to provide a large class of examples of slowly decreasing functions :

Proposition 1. Let $\mu, f, \Omega, K$ be as above. Assume:

i ) $V=V(f)=\left\{\left(z_{k}, m_{k}\right)\right\}$ is $(\Omega+K)$-interpolating;

ii) the multiplicities $m_{k}$ are bounded by an integer $m$;

iii) $f$ is of completely regular growth;

iv) the zeros of $f$ form an $R$-set (see [4], page 95), in the sense that there exists $d>0$ such that the circles of centers $z_{k}$ and radii

$$
r_{k}=d\left|z_{k}\right|^{1-\left(\left|z_{k}\right| / 2\right)}
$$

do not intersect.

Then, $\forall \varepsilon>0 \quad \exists C_{\varepsilon}>0$ such that

$$
|f(z)| \geqq C_{\varepsilon}(d(z, V))^{m} \exp \left(H_{K}(z)-\varepsilon|z|\right) .
$$

Proof. From Lemma 2, and by ii), we deduce that (6) holds in sufficiently small circles $\Delta_{k}$ around the points $z_{k}$, with fixed radii, and with a uniform value for the constant $C_{\varepsilon}$ (this follows from the mean value theorem, the growth conditions on $f$, and the fact that $H_{K}(z)$ changes little in nearby points). On the other hand, in the proof of the theorem 5 of chapter II of [4] (see page 127), it is shown that: $\forall \varepsilon>0 \exists r_{\varepsilon}>0$ such that, $\forall r>r_{\varepsilon}$,

$$
\ln \left|f\left(r \cdot e^{i \theta}\right)\right|>\left[H_{K}\left(e^{i \theta}\right)-\varepsilon\right] r,
$$

for all $z=r e^{i \theta}$ outside the circles described in iv). Therefore, since these circles can be taken smaller than the $\Delta_{\dot{k}}$ in which (6) already holds (due to 
their decreasing radii), and since (6) certainly holds in $\left\{|z|\left\langle r_{\varepsilon}\right\} \backslash \cup \Delta_{k}\right.$ (a compact set where $f$ never vanishes), we obtain the proposition.

\section{§3. Fabry Type Theorems}

In this last section we provide the main results of our paper. We employ the notations of Section $2:$ in addition, for $\theta \in(0, \pi / 2)$, let $\Gamma_{\theta}=$ $\{z \in C: \pi / 2-\theta<\arg z<\pi / 2+\theta\}$ and denote by $\tilde{\Gamma}_{\theta}$ the open set such that $\Gamma_{\theta}=\tilde{\Gamma}_{\theta}+K$, so that $\mu *: \mathscr{G}\left(\Gamma_{\theta}\right) \rightarrow \mathscr{H}\left(\tilde{\Gamma}_{\theta}\right)$.

We begin by proving a preliminary lemma in the same spirit as the hyperbolicity results proved in [6]:

Lemma 3. Let $\mu \in \mathscr{H}^{\prime}(\mathbb{C})$ be slowy decreasing. Suppose that $\mu$ satisfies the following condition:

$\left(C_{\theta}\right) \quad \exists \varepsilon>0$ such that (with at most a finite number of exceptions), all $z \in V \cap\{\operatorname{Im} z<0\}$ do not belong to $\{z \in C: \pi+\theta-\varepsilon<\arg z<2 \pi-\theta+\varepsilon\}$.

Then, $\forall a \in \boldsymbol{R}^{+}$, every solution $f \in \mathscr{H}\left(\Gamma_{\theta}-i a\right)$ of $\mu * f=0$ extends to a solution $\tilde{f} \in \mathcal{H}\left(\Pi_{+}-i a\right)$ of the same equation.

Proof. Since $\mu$ is slowly decreasing and $\mu * f=0$, the theorem 7 of [6] shows that $f$ can be represented by a series, convergent on $\Gamma_{\theta}-i a$, of the form

$$
f(z)=\sum_{k=1}^{+\infty}\left(\sum_{i=1}^{J_{k}} P_{k, \jmath}(z) \exp \left(\alpha_{k, \jmath} \cdot z\right)\right),
$$

with $P_{k, j}$ polynomials, and $\hat{\mu}\left(\alpha_{k, j}\right)=0$. Therefore if on $V$, the weights which describe the topologies of $\hat{\mathscr{H}}^{\prime}\left(\Gamma_{\theta}-i a\right)$ and of $\hat{\mathscr{H}}^{\prime}\left(\Pi_{+}-i a\right)$ are comparable, one deduces that the series is, actually, convergent in $\mathcal{H}_{(}\left(\Pi_{+}-i a\right)$; of course this is still true if a finite number of zeros do not satisfy this requirement. This comparison of the topologies can be rephrased as follows: for any compact $\tilde{T} \subset\left(\Pi_{+}-i a\right)$, there exists a compact $T \subset\left(\Gamma_{\theta}-i a\right)$ such that, for all $z \in V$, it is true that

$$
H_{\tilde{T}}(z) \leqq H_{T}(z) .
$$

Clearly this condition does not impose any requirement on the $z \in V$ for which $\operatorname{Im} z>0$; on the other hand, by observing that $H_{K}(z)$ is just the supremum (for $\xi \in K$ ) of the scalar product of $z$ and $\xi$, one can easily check that the only problems in fulfiling (7) are created by those $z$ which lie on the normals to $\arg z=\pi / 2 \pm \theta$. Thus, due to our hypotheses, the result follows.

We are now ready to prove our main results : 
Theorem 1. Let $\mu \in \mathscr{H}^{\prime}(\boldsymbol{C})$ be slowly decreasing, and suppose it satisfies $\left(C_{\theta}\right)$. Let $f \in \mathscr{K}\left(\Gamma_{\theta}\right)$ be a solution of $\mu * f=0$. If there exists a neighborhood $v$ of the origin to which $f$ extends holomorphically, then we can find $\delta>0$ such that $f$ extends holomorphically to $\tilde{f} \in \mathfrak{H}\left(\Pi_{+}-i \delta\right)$, which, there, satisfies $\mu * \tilde{f}=0$.

Proof. By Lemma 3, $f$ can be extended to $f_{1} \in \mathscr{H}\left(\Pi_{+}\right), \mu * f_{1}=0$. Choose now $\theta^{\prime} \in(0, \pi / 2)$, and $\delta>0$ in such a way that:

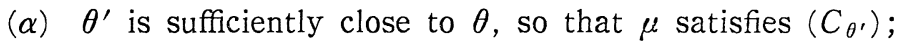

( $\beta)\left(\Gamma_{\theta},-i \delta\right) \subset \Pi_{+} \cup \mathcal{U}$.

Then, by $(\beta)$ and analytic continuation, $f_{1} \in \mathscr{K}\left(\Gamma_{\theta^{\prime}}-i \delta\right)$, and it satisfies, in $\Gamma_{\theta},-i \delta, \mu * f_{1}=0$. By $(\alpha)$, we can again apply the lemma to extend $f_{1}$ to $\tilde{f} \in \mathscr{K}\left(\Pi_{+}-i \delta\right)$, which solves $\mu * \tilde{f}=0$.

Remark 9. This result provides a two-fold extension of Ehrenpreis' approach. Indeed, not only we have an analytic continuation result for series of the form $\sum_{j=1}^{+\infty} a_{\jmath} \exp \left(i \cdot b_{j} \cdot z\right)$ which arise as solutions of suitable convolution equations, but we also proved the same result for the more complex series $\sum_{k=1}^{+\infty} \sum_{j=1}^{J k} P_{j, k}(z) \exp \left(i \cdot a_{k} \cdot z\right)$. Notice that, in our treatment, the lacunarity condition of the Fabry gap theorem is replaced by the slow decrease assumption on $\mu$ : both of these requirements imply that the roots of $\hat{\mu}$ cannot be too close to one another.

For our next result, which provides an extension of Kawai's main result in [3], we consider, for a sequence $\left\{z_{n}\right\}$ of complex numbers, the following conditions :

i) $z_{n} \neq 0, \lim _{n \rightarrow \infty} n /\left|z_{n}\right|=0$;

ii) $\exists C>0$ such that, $\forall m, n$ integers,

$$
\left|z_{n}-z_{m}\right| \geqq C|\imath \imath-m| ;
$$

iii) there exist finitely many unit vectors $e_{k}(k=1, \cdots, t)$ in $S^{1} \equiv \mathbb{R}^{2}$ for which the following holds:

$$
\begin{aligned}
& \forall \varepsilon>0, \text { and for each compact } \\
& K \subset S^{1}-\left\{e_{1}, \cdots, e_{t}\right\}, \exists n_{0}=n_{0}(\varepsilon, K)
\end{aligned}
$$

such that

$$
\inf _{\substack{n \geqq n \\ e \in K^{0}}}\left|\frac{\left(\operatorname{Re} z_{n}, \operatorname{Im} z_{n}\right)}{\left|z_{n}\right|}-e\right|>\varepsilon
$$

Remark 10. Conditions (i) and (ii) are well known, and guarantee that the infinite product $\Pi_{n=0}^{+\infty}\left(1+\left(\zeta^{2} / z_{n}^{2}\right)\right)$ is a well defined entire function $P(\zeta)$ such that $P(d / d t)$ is a linear differential operator of infinite order (see e.g. [5], theorem XXXI and [8], chapter II, §1.4). On the other hand, condition (iii) is due to 
Kawai [3], introduced in order to get hold of the characteristic directions, for $P(d / d z)$, and to apply to it the microlocal invertibility results of [8].

Theoren 2. Let $\mu \in \mathscr{H}^{\prime}(C)$ be slow'y decreasing and such that condition $\left(C_{\theta}\right)$ is satisfled for a countable set of points $\left\{z_{n}\right\}$ which satisfy conditions (i). (ii), (iii) above. Then if $f \in \mathscr{H}\left(\Gamma_{\theta}\right)$ satisfy $\mu * f=0$, and if there exists $\mathcal{U}$, neighborhood of the origin, to which $f$ exiends holonorphically, then there exists $\delta>0$ such that $f$ extends to $\tilde{f} \subseteq \mathcal{H}\left(\Gamma_{\theta}-i \delta\right)$ which satisfies $\mu * \tilde{f}=0$.

Proof. From the hypotheses, one deduces the existence of $\mu_{1} \in \mathscr{H}^{\prime}(\mathcal{C})$, satisfying the hypotheses of one Theorem 1, and of a partial differential operator of infinite order $P(d / d z)$ (as in Theorem 1 of [3]), such that $\mu_{1} * P(d / d z) f=0$. By Theorem 1. $P(d / d z) f=g$ extends, for some $\delta_{1}>0$, to $\check{g} \in \mathscr{H}\left(\Pi_{+}-i \delta_{1}\right)$; let now $u \in \mathscr{H}\left(\Pi_{+}-i \delta_{1}\right)$ be such that $P(d / d t) u=\hat{g}$ (such $u$ exists by the surjectivity of $P(d / d z)$, see, e. g. [6], the remark after Theorem 1;。 Then $P(d / d z)(f-u)=0$ on $\Gamma_{\theta}$ and so, by [3]. Theorem $1, f-u$ extends to some $\tilde{u} \in \mathcal{H}\left(\Gamma_{0}-i \delta_{2}\right)$, for some $\delta_{2}>0$. If now $\delta=\min \left(\delta_{1}, \delta_{2}\right)$, the statement follows with $\tilde{f}=u+\tilde{u}$.

Remark 11. It should be clear that the tools we have used have not much to do with the fact that we are working in only one variable. Indeed one might easily extend Theorems 1,2 to several variables. In this case, of course, a series is not going to be the general solution of a single convolution equation. but one has to deal with systems $\mu_{1} * f=\cdots=\mu_{n} * f=0, \mu_{,} \in \mathscr{H}^{\prime}\left(\mathbb{C}^{n}\right)$, and Definition 1 must be replaced by the notion of "joint slow decrease", as given in [6]. Generally speaking, this variation will make more difficult the task of Providing concrete examples to which Theorems 1 and 2 apply (in particular, Proposition 1 ceases to be true), but from a conceptual point of view, nothing new would take place in Section 3.

\section{References}

1. Berenstein, C.A. and Taylor, B. A., A new look at interpolation theory for entire functions of one variable Advances in Math., 33 (1979), 109-143.

- 2] Ehrenpreis, L., Fourier Analysis in Several Complex Variables. Wiley-Interscience, New York, 1970.

3 1 Kawai, T., The Fabry-Ehrenpreis gap theorem and linear differential equations of infinite order, RIMS preprint 484, Kyoto Univ., Kyoto, 1984.

-4] Levin, B. Ja., Distribution of Zeroes of Entire Functions, Amer. Math. Soc., Providence, R.I., 1964.

[5] Levinson, N., Gap and Density Theorems, Amer. Math. Soc., New York, 1940.

[6] Meril, A. and Struppa, D. C., Convolutors in spaces of holomorphic functions, Proc. Spec. Year Complex Analysis, University of Maryland, 1985/86, to appear.

[7] Morzhakov, V.V., Convolution equations in spaces of functions holomorphic in convex domains and on convex compacts in $\mathbb{C}^{n}$, Mat. Zam., 16 (1974), 431-440. 
[8] Sato, M., Kawai, T. and Kashiwara, M., Microfunctions and pseudodifferential equations, Lecture Notes in Math., 287, Springer, Berlin-Heidelberg-New York, 1973 , pp. $265-529$.

[9] Squires, W.A., Interpolation theory for spaces of entire functions with growth conditions, Ph.D. thesis, Univ. of Michigan, 1980.

[10] Yger, A., Inégalités de Lojasiewiez ou de Malgrange pour certaines familles d'elements de $\hat{g}^{\prime}(\boldsymbol{R})$, manuscript, 1986. 